\title{
Boletus Queletii Schulzer in Parainen, SW-Finnland
}

\author{
Seppo Hietavuo
}

Schon lange hat man gewusst (KARSTEN 1859), dass die Eichenwälder im Südwesten Finnlands, besonders in Ruissalo (Turku) viele Pilz-Arten haben, die die Flora der klimatisch günstigeren Gebiete Mittel-Europas repräsentieren (vgl. auch Kallio 1963). Weil Parainen, eine Insel-Gemeinde etwa $20 \mathrm{Km}$ von der Stadt Turku entfernt, neben seinem günstigen Klima noch Kalk in seinem Boden birgt, dürfte die geringe Untersuchung als Ursache sein, dass dieser von seinem Eichenwäldern bekannte Ort noch nicht ein ebenbürtiger Konkurrent mit den Eichenwäldern Ruissalos geworden ist. Einige von den südlichen Pilzarten von Parainen sind doch in der Liste von Kallio 1963 sowohl von Kallio \& Kankainen 1967 mitgenommen. 1965 (und danach jährlich) habe ich hier Boletus Queletii Schulzer 1885 (Syn. Boletus erythropus Pers., non Fr., 1796) gefunden.

B e s c h re i bung (verkürzt nach Kallenbach 1927 , S. 21-22):

Hut anfangs meistens olivgelb bis bräunlich, dann \pm rötliche Töne; orange, ziegelrot bis dunkel karminblutrot, in allen möglichen Kombinationen und Variationen; im Alter wieder fahler; gegen Druck \pm empfindlich; fein filzig und daher öfter samtigschillernd.

Fleisch \pm gelb; unter Huthaut, von Stielrinde und -basis her \pm wein- bis blutrot eindringend; Röhrenansatz gelb; \pm blauend; fest.

Röhren blass zitrongelblich, allmählich stärker bis goldgelblich, schliesslich mehr oliv; bei Verletzung grünblau; um Stiel \pm eingebuchtet.

Poren allmählich goldgelblich und mit rötlichem Hauch, dann schön und leuchtend orangerot, bald bis oliv; bei Druck \pm stark blauend.

Stiel sehr veränderlich in Form und Färbung; olivgelb, allmählich lebhafter, zitron- bis goldgelb; von Basis her blutkarminrot; \pm feinfilzig-punktiert; immer ohne Spur von Adernetz; Druck- stellen blauend, anfangs eiförmig-knollig-bauchig, allmählich \pm keuliggestreckt.

Chemische Reaktionen. Säuren: Nach 1 Tag mit den saueren Reagentien alles viel leuchtender: gelb, orange und rot herrschen vor. Mit basischen Reagentien viel trüber, uberall mit oliv.

Sporen gedrungen, verschieden in Form und Grosse, (8) $10-14$ (17) /5-7 (9) $\mu$.

Verwechslungsmöglichkeiten in Finnland:

In unserem Lande hat man früher drei Arten von der rotsporigen sog. Luridus-Gruppe gefunden: Boletus miniatoporus Secr. (B. erythropus Fr. non Pers) unterscheidet sich von den übrigen durch den meistens dunklen Hut, die düster mennig-blutroten Poren, das sattgelbe, viel rascher und stärker blauende Fleisch und die auffallende Filzpunktierung des Stieles (Kallenbach 1927, S. 22). Auch ist $B$. miniatoporus \pm unabhängig von Kalk (ANDERsson) während B. Queletii wenigstens bei seiner Nordgrenze Kalk fordern dürfte. Noch leichter ist es B. Queletii von Boletus luridus (Schaeff.) Fr. zu unterscheiden, das immer den genetzten Stiel hat wie auch von Boletus satanas Lenz aus demselben Grund. Auch B. rhodoxanthus, das in Finnland noch unbekannt ist, gehört $\mathrm{zu}$ diesem genetzten Typus. Von diesen fordert B. luridus nicht unbedingt Kalk (ANDERSSON).

Vorkommen in Europa:

Dänemark: Ziemlich selten. Deutschland: Nicht häufig. England: Nur var. lateritius, mit anfangs gelben Poren, selten. Auch in Frankreich, Italien, Jugoslavien, Österreich, der Schweiz, Ungarn und Nordafrika (KalLenbach S. 22-23, Friesta I-VII, Pearson). Schweden und Norwegen: Mir nicht bekannt. 
Die Kenntnisse von der Verbreitung dieses Pilzes sind leider alt und mangelhaft.

\section{Standort :}

Nach Kallenbach (S. 22) 'Ränder lichter Laubhochwälder, Buchen- und Eichenwälder, oft an den Böschungen der Waldstrassen von Anfang Juni bis September; später (bis Anfang Oktober bei milder Witterung) tritt er seltener auf; meistens einzeln, seltener in Büscheln (bis zu ungefähr 5 Pilze verwachsen). Öfters auf dem nackten Boden, aber auch zwischen Laub und Gras.'

Selbst fand ich diesen Pilz 31.8.1965 in Peksor, Parainen, auf einer westgeneigten lehmigen offenen Stelle, mit starker Neigung. In der Nähe wuchs ein Eichenspross, weiter Eichen und Birken. Auf jenem Platz gab es 2 Fruchtkörper. Der Standort ist alkalisch ( $\mathrm{pH}$ 7.2). Die Sporen entsprechen den Werten von KallenBach, ihre Durchschnittsgrösse war $13.66 \times 4.49 \mu$ (200 St.). Auf demselben Platz wuchs 7. September noch 1 Ex., danach habe ich die Art nicht dort gesehen. Nahe bei diesem Platz habe ich folgende seltene Pilze gefunden:

Boletus versicolor Rostk. (Syn. B. rubellus. Krombh. B. barlae Krombh., B. sanguineus Fr.), Suillus granulatus und Xerocomus chrysenteron. R. Lundqvist hat dort auch Fistulina hepatica gefunden. (Von B. versicolor gibt es Proben sowohl im Bot. Museum der Universität Turku als bei mir.).

Den anderen Standort fand ich in Lenholm, Parainen 30: 8. 1966. Zwei Exemplare wuchsen auf einem dünnen, alten Strohhaufen an der Grenze zwischen einem Eichenhain und einem Acker, auf einem südexponierten Standort. Der eine wuchs unter einer grossen Eiche, der andere neben einer kleinen Fichte (4 Meter von jener Eiche). Auf dem Platze wuchsen u.a. auch einige Birken, ein Kiefer, Anemone nemorosa, Hepatica tritoba, Urtica dioeca, Filipendula ulmaria, Geum rivale. In der Entfernung von nur 10 Metern geht der Hain zu trockenem, kargem Kiefernwald über. $\mathrm{pH}$ liegt zwischen 6 und 7. Auf demselben Platz wächst auch Xerocomus chrysenteron und in seiner Nähe Suillus granulatus. 3. 9. 1967 fand ich dort 5 Fruchtkörper (nicht in Büscheln, vgl. Kallensach) und 4.9. 1968 (da es sehr wenige andere Pilze gab) wieder $2 \mathrm{Ex}$.

3.9. 1967 fand ich 2 Fruchtkörper auf einem dritten Standort, etwa $1 \mathrm{Km}$ östlich vom zweiten. Dieser Platz ist eine warme und trockene Wiese, wo da und da grosse Eichen und Linden, kleine Fichten und viele Wacholder wachsen, 50 Meter vom Meeresufer. Beide wuchsen nahe bei den kleinen Fichten, 4 und 9 Meter von den grossen Eichen, etwa 30 Meter von einander. $\mathrm{pH}$ ist 6,4. Da Wuchs auch $X$. chrysenteron. 4. 9. 1968 gab es 4 Ex. von B. Queletii, in einer Haufe.

Von B. Queletii gibt es nun 4 Proben, 2 im Bot. Museum der Universität Turku und 2 bei mir. Beide haben eine Probe von Peksor 1965 und eine von Lenholm 1966.

Boletus Queletii ist also offenbar eine sowohl klimatisch als edaphisch (Kalk) anspruchsvolle Art (wie z. B. Boletus satanas Lenz.), die doch auch in anderen Kalkstandorten ( $\mathrm{z}$. B. Lohja?) des Eichengebietes zu finden sein dürfen.

\section{LITERATUR}

Andersson, O., 1943: Studier över Boletaceer. Bot. Not., 185-202.

Kallenbach, F., 1927: Die Pilze Mitteleuropas. Bd. I. Die Röhrlinge (Boletaceae), 21-22.

Kallio, P., 1963: Zur Verbreitung einiger in Finnland südlichen Pilze, besonders in der südwestlichen Eichenzone. - Karstenia VIVII, 35-76.

Kallio, P. \& E. Kankainen, 1967: Contributions to the macromycetes in the oak zone of Finland. - Karstenia VIII, 9-13.
Karsten, P., 1859: Sydvestra Finlands Polyporeer. - Akad. Afhandl., 47 p. Helsinki.

LANGe, M., 1961: Illustreret Svampeflora. Köbenhavn.

Luther, H. \& I. Luther, 1965: Ett fynd av Boletus miniatoporus Secr. in Tvärminne. Memoranda Soc. F. Fl. Fenn. 41, 31-36.

Pearson, A. A., 1946: British Boleti. New Naturalist, $1-19$. 